\title{
Calcified Abdominal aortic occlusion: Treatment using Intravascular Lithotripsy
}

\author{
Pratik Shah $^{1}$ Vimal Someshwar ${ }^{1}$ Krishna Mundada ${ }^{2}$ Abhijit Raut ${ }^{1}$ \\ ${ }^{1}$ Department of Radiology, Kokilaben Dhirubhai Ambani Hospital and \\ Medical Research Institute, Mumbai, Maharashtra, India \\ 2 Dr. R.N. Cooper Hospital, Mumbai, Maharashtra, India \\ Address for correspondence Krishna Mundada, Final Year MBBS, H.B. \\ T. Medical College and Dr. R.N. Cooper Hospital, JVPD Scheme, Juhu, \\ Mumbai, 400056, Maharashtra, India \\ (e-mail: mundadakrishnavinod29@gmail.com).
}

J Clin Interv Radiol ISVIR 2022;6:141-143.
Abstract
Keywords
- abdominal aortic calcification
- IVL technique
- IVUS

Near total occlusion of abdominal aorta due to atherosclerotic calcification is a challenging condition to treat. Though use of intravascular lithotripsy (IVL) has proven to be an effective technique in the treatment of calcified arterial lesions, the data on the use of the technique in high grade occlusion of aortic stenosis is still limited. We present a case of abdominal aortic calcification treatment in a patient using IVL technique.

\section{Introduction}

An 80-year-old female, past smoker, with a history of bilateral Iliac stenting 15 years back, came to hospital outpatient department with severe limb claudication in both lower limbs. The limb pain got worse over the course of months and occurred even when she walked for short distances. Examination revealed absent femoral and lower limb pulsations.

Occlusion of bilateral common iliac artery stents was suspected and computed tomography (CT) angiogram was performed that showed dense calcification of the abdominal aorta and bilateral iliac arteries. There was total occlusion over a short segment in the mid-infrarenal aorta, which was confirmed on dual-source CT angiogram. Bilateral iliac stents were patent with good distal runoff in lower limb vessels (-Figs. 1 and 2).

An access was taken from left brachial artery. The abdominal aortic angiogram showed severe narrowing of infrarenal aorta, diameter being $10 \mathrm{~mm}$, and patent stents in both iliac arteries ( - Fig. 3A). ProGreat (Terumo) microcatheter was used to cross occluded segment in the aorta. Over Fielder FC wire, predilatation was done with 4.0 X $60 \mathrm{~mm}$ Armada balloon. Access was also taken through right femoral artery and 9 Fr. Mullin Cook sheath was introduced. Intravascular ultrasound (IVUS) was performed across stenotic segment using $8.5 \mathrm{Fr}$. Ultra ICE plus (Boston Scientific, Marlborough, Massachusetts, United States) probe, which confirmed severe narrowing due to hard nodular atherosclerotic wall calcification. Post-IVUS, 6.5 x $60 \mathrm{~mm}$ shockwave intravascular lithotripsy (IVL) catheter was taken across stenotic segment, with parallelly placed $4 \mathrm{~mm} \times 60 \mathrm{~mm}$ balloon, to achieve apposition of the IVL balloon to the wall of the aorta. Lithotripsy was performed by rotating the two balloons along its long axis (- Fig. 4A). Post-IVL, IVUS showed successful fracture of the calcification of the aortic wall, which appeared homogenous and cloudy with improved flow across stenotic segment (-Fig. 4B). Omnilink balloon mounted stent $(9 \times 59 \mathrm{~mm})$ was placed across stenotic segment of infrarenal abdominal aorta. Final angiogram showed good flow across aortic and iliac stents (-Fig. 5). At 6-month follow-up, the patient has no symptoms of intermittent claudication and pain in lower limbs. received

April 10, 2021

accepted

June 9, 2021

published online

February 17, 2022
DOI https://doi.org/

10.1055/s-0041-1732797. ISSN 2457-0214. (c) 2022. Indian Society of Vascular and Interventional Radiology. All rights reserved.

This is an open access article published by Thieme under the terms of the Creative Commons Attribution-NonDerivative-NonCommercial-License, permitting copying and reproduction so long as the original work is given appropriate credit. Contents may not be used for commercial purposes, or adapted, remixed, transformed or built upon. (https://creativecommons.org/ licenses/by-nc-nd/4.0/)

Thieme Medical and Scientific Publishers Pvt. Ltd., A-12, 2nd Floor, Sector 2, Noida-201301 UP, India 


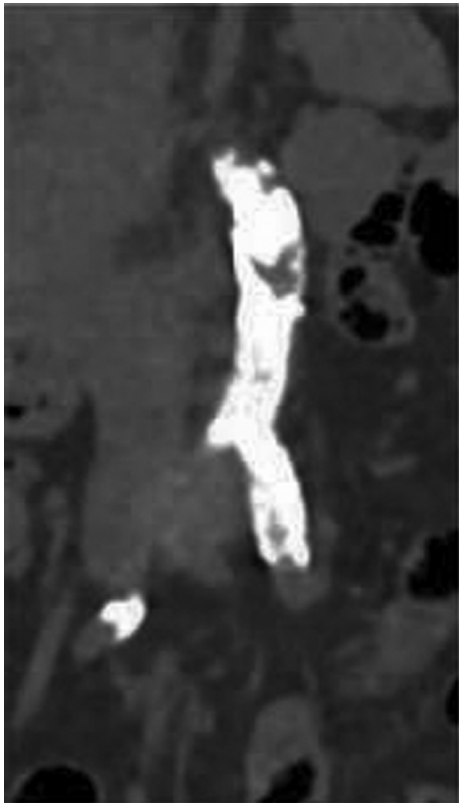

Fig. 1 Plain computed tomography of abdominal aorta suggestive of diffuse atherosclerotic wall calcification.

\section{Discussion}

Abdominal aortic calcification is strongly associated with smoking in general population. A study by Jung et al suggest even a low level of daily smoking increases the likelihood of aortic calcification; similar conclusion was shown from 5year follow-up study by Pham et al. ${ }^{1,2}$ Atherosclerotic plaque calcification usually affects the intimal layer of the wall, while calcification in the medial layer is ascribed to hypophosphatemia and hypercalcemia. The patient in our report is a smoker for over 20 years without any other comorbidities that are known to cause the condition. ${ }^{3,4}$

Treatment of severe aortic calcification involves challenging maneuvers and careful technique to avoid various complications like dissection, perforation, and formation of emboli or unsuccessful reperfusion. ${ }^{5}$ It is difficult to perform balloon angioplasty in these patients as effective dilatation and revascularization are not achieved. The patient in our report had occlusion of the lumbar aortic segment. Given the extensive calcification of the aorta, it was difficult to negotiate a guidewire across the calcified plaque and decide the type of balloon angioplasty catheter to achieve an adequate dilatation.

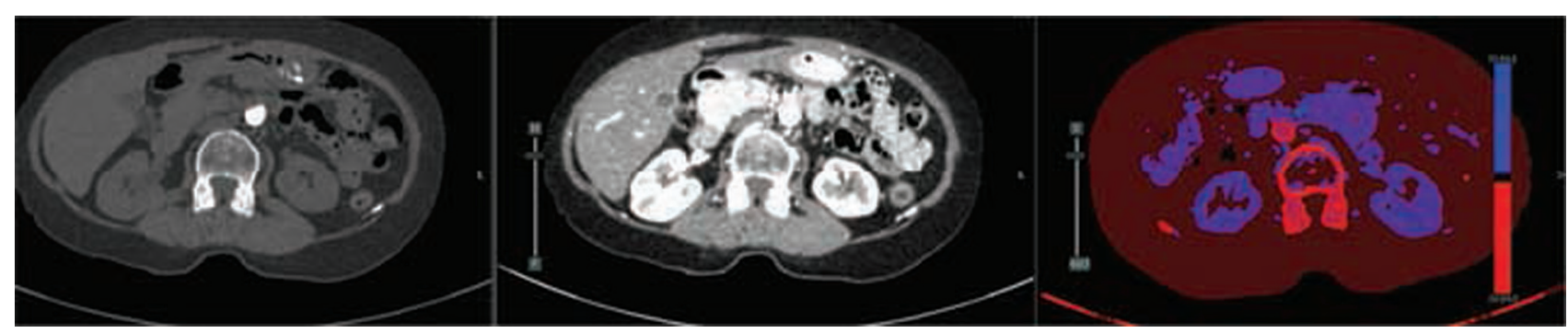

Fig. 2 (A) Axial plain computed tomography (CT) scan, (B) contrast angiogram, and (C) Dyna CT images suggestive of occlusion of infrarenal
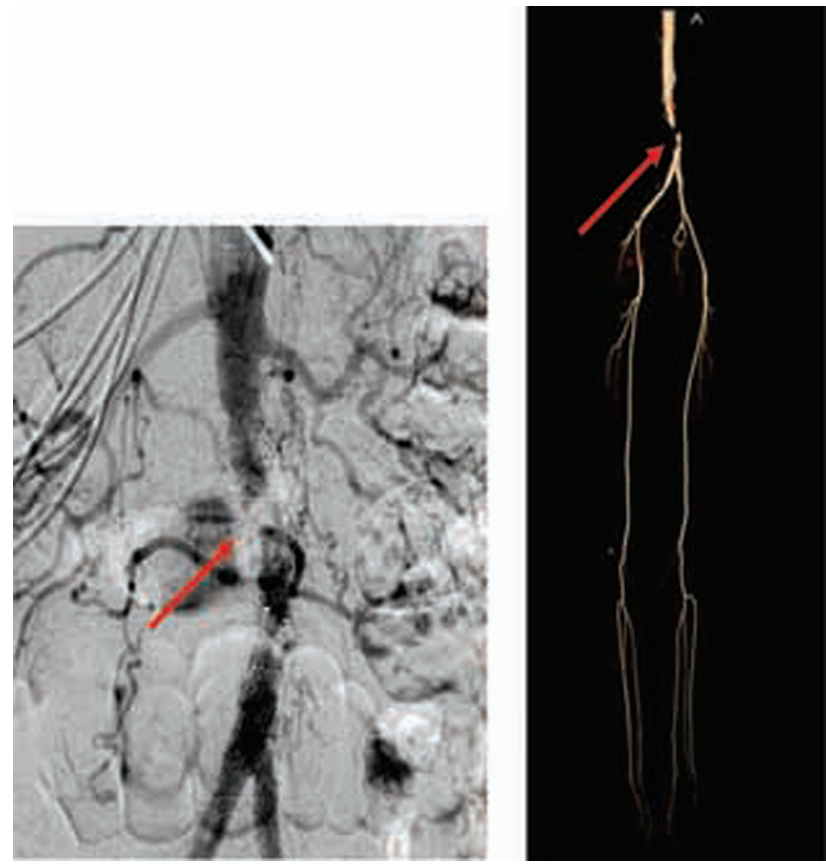

Fig. 3 (A) Digital subtraction angiography abdominal angiogram and (B) reconstructed image of computed tomography angiogram suggestive of severe stenosis of infrarenal abdominal aorta.
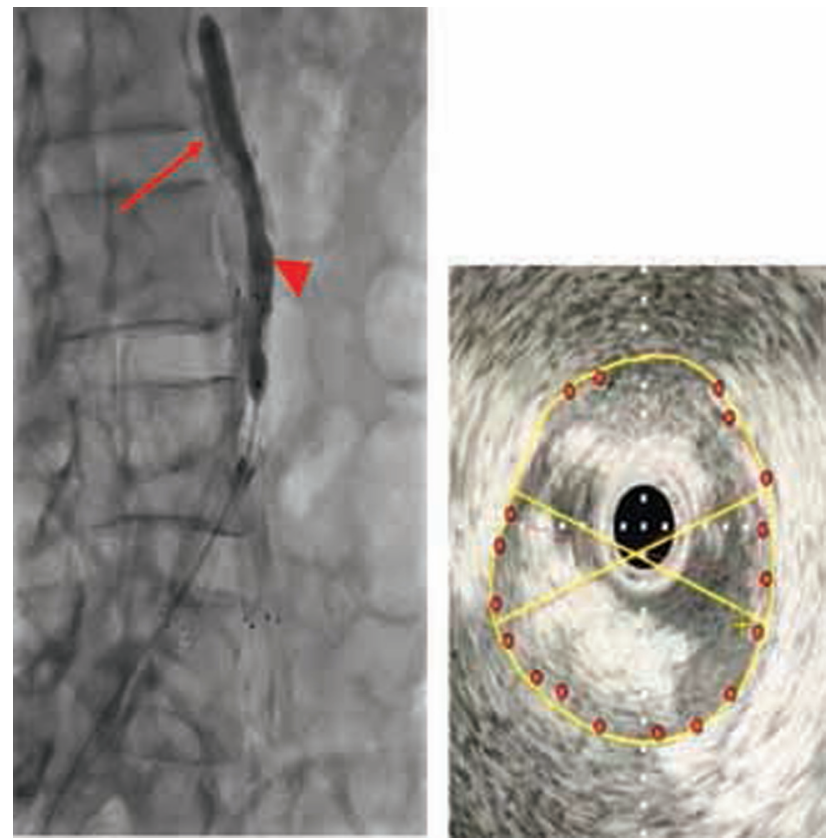

Fig. 4 (A) Double balloon technique (arrow showing $4 \times 60 \mathrm{~mm}$ balloon, and arrowhead pointing toward $6.5 \times 60 \mathrm{~mm}$ lithotripsy balloon). (B) Intravascular ultrasound showing fractured calcification at the same level. 


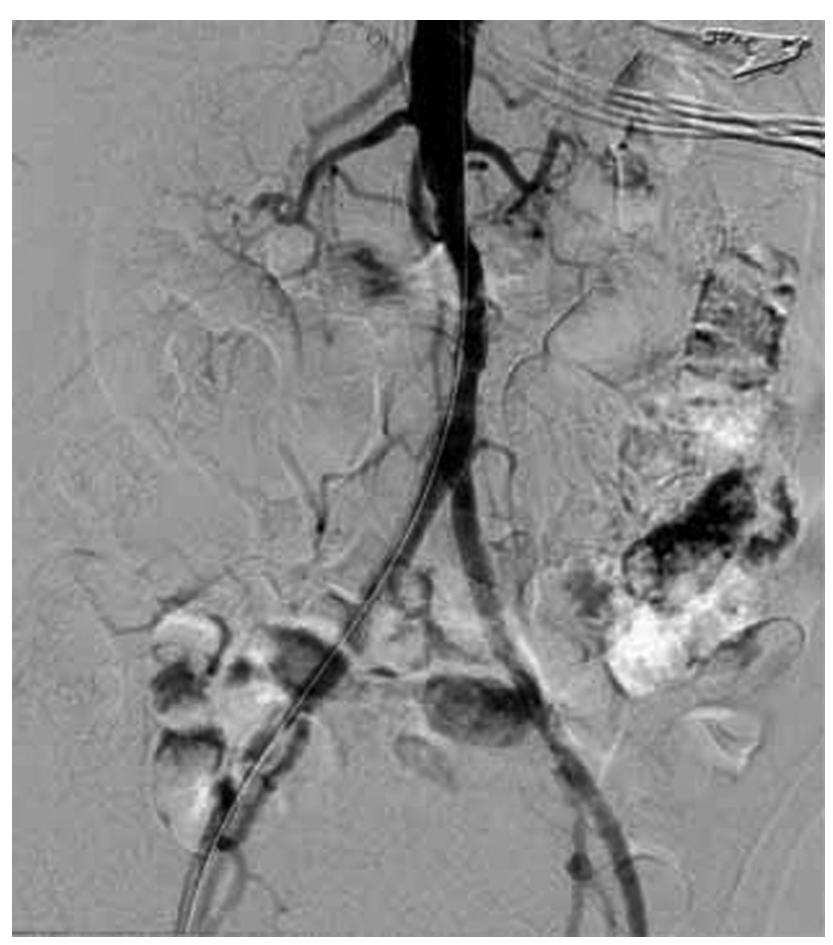

Fig. 5 Postprocedural angiogram showing good flow across infrarenal abdominal aorta and both the iliac stents.

IVL is a novel technique in the treatment of arterial calcification and has shown to be effective in patients with severe calcifications. ${ }^{5-7}$ The technique has been used successfully in the treatment of various large diameter arteries like the aorta, femoral, and popliteal calcification. The technique relies on the use of shockwaves for breaking down calcific deposits on vessel wall, similar to urological lithotripsy. It involves delivery of 30 pulses at a rate of 1 pulse per second for a maximum of 10 cycles via the catheter. Literature search shows use of similar double-balloon technique and IVL for successful treatment of juxtarenal coral reef aorta. ${ }^{8}$ The use of the technique in our patient also resulted in successful breakdown of calcification and softening of the wall of the aorta. Post-IVL angiogram confirmed good apposition of the stent and improved flow in the lower limb arteries.

IVL is thus a promising technology in the management of aortic steno-occlusion due to calcified atherosclerotic plaques.

\section{Conflict of Interest}

None declared.

\section{References}

1 Jung JG, Wu LT, Kim JS, Kim ED, Yoon SJ. Relationship between smoking and abdominal aorta calcification on computed tomography. Korean J Fam Med 2019;40(04):248-253

2 Pham T, Fujiyoshi A, Hisamatsu T, et al.Shiga Epidemiological Study of Subclinical Atherosclerosis (SESSA) Research Group. Smoking habits and progression of coronary and aortic artery calcification: a 5-year follow-up of community-dwelling Japanese men. Int J Cardiol 2020;314:89-94

3 prpt . Sethi A, Taylor L. Ruby JG, et al. Calcification of abdominal aorta is a high risk underappreciated cardiovascular disease factor in a general population. MedRxiv, January 1, 2020, 2020.05.07.20094706. https://doi.org/10.1101/2020.05.07. 20094706./prpt

4 Bartstra JW, Mali WP, Spiering W, de Jong PA. Abdominal aortic calcification: from ancient friend to modern foe. Eur J Prev Cardiol 2020;2047487320919895:2047487320919895Epub ahead of print. Doi: $10.1177 / 2047487320919895$

5 Adams G, Shammas N, Mangalmurti S, et al. Intravascular lithotripsy for treatment of calcified lower extremity arterial stenosis: initial analysis of the disrupt PAD III study. J Endovasc Ther 2020; 27(03):473-480

6 Brodmann M, Werner M, Holden A, et al. Primary outcomes and mechanism of action of intravascular lithotripsy in calcified, femoropopliteal lesions: results of disrupt PAD II. Catheter Cardiovasc Interv 2019;93(02):335-342

7 Brodmann M, Werner M, Brinton TJ, et al. Safety and performance of lithoplasty for treatment of calcified peripheral artery lesions. J Am Coll Cardiol 2017;70(07):908-910

8 Chag MC, Thakre AA. Novel use of intravascular lithotripsy for coral reef aorta: a case report. Eur Heart J - Case Reports 2021;5 (04):ytab102https://doi.org/10.1093/ehjcr/ytab102 